\title{
Composite solid lipid nanoparticles with hydrogel core for delivering small hydrophilic molecules to the retina: an in vitro study using ARPE-19 and 661W cells.
}

\author{
Erico Himawan ${ }^{\mathrm{a}, 1, *}$, Li Huang ${ }^{\mathrm{b}, 1}$, Soumaya Belhadjc, Raul Oswaldo Perez Garcia ${ }^{\mathrm{d}}$, \\ François Paquet Durand ${ }^{c}$, Nicolaas Schipper ${ }^{\mathrm{d}}$, Matej Buzgo ${ }^{\mathrm{a}}$, Aiva Simaite ${ }^{\mathrm{a}}$, Valeria \\ Marigo $^{\mathrm{b}, *}$ \\ ${ }^{a}$ InoCure s.r.o, Research and Development Department, Prague, Czech Republic \\ ${ }^{b}$ Department of Life Sciences, University of Modena and Reggio Emilia, Italy \\ 'Institute for Ophthalmic Research, Cell Death Mechanism Group, University of Tübingen, Germany \\ ${ }^{d}$ Division Bioeconomy and Health-Chemical Processes and Pharmaceutical Development, Research \\ Institute of Sweden, Sweden
}

${ }^{1}$ These authors equally contributed to this work.

${ }^{*}$ Corresponding authors:

email addresses: $\underline{\text { erico@inocure.cz, }}$ valeria.marigo@unimore.it

\begin{abstract}
Aim: To formulate a solid lipid nanoparticle (SLP) containing hydrogel core and composite shell for delivering small hydrophilic molecule to the retina. Methods: SLP was prepared using modified water-in-oil-in-water emulsion technique. Cellular uptake of the SLP were evaluated in vitro using ARPE-19 and 661W cells. Results: SLP encapsulation of a neuroprotective cGMP analogue resulted in a particle size $<250 \mathrm{~nm}$, anionic charge $>-20 \mathrm{mV}$, and encapsulation efficiency value of $60 \%$. SLP hydrogel core and composite shell improves encapsulation efficiency and polydispersity, respectively. In vitro studies showed a successful uptake and release of the hydrophilic cargo inside the cells. Conclusion: Composite SLP with hydrogel core could be useful in developing retinal disease treatment using a small hydrophilic active molecule.
\end{abstract}

\section{Key words}

661W; ARPE19; cGMP; drug delivery system; solid lipid nanoparticle.

\section{Background}

Retinal degeneration relates to different progressive diseases leading to vision loss, including diabetic retinopathy [1], age-related macular degeneration [2], and hereditary retinal degeneration [3]. Together, these diseases constitute an important health challenge that severely affect the quality-of-life of patients and has a significant socio-economic impact [4]. The cell types typically affected by these diseases are photoreceptors and retinal pigment epithelium (RPE) cells. While several different drugs have been proposed for treatment development, a key challenge is the delivery of an active pharmaceutical ingredient to the target cells within the retina and then maintain a large enough drug concentration over a prolonged period. This difficulty is exacerbated by the access-limiting blood retinal barrier. In this paper, we present a solid lipid nanoparticle (SLP) formulation with a composite shell and a hydrogel core that may be used as retinal drug delivery system (DDS).

In current clinical practice intravitreal injections are routinely used to administer treatments to the retina. This route allows DDS to bypass several ocular barriers like the corneal epithelium, the conjunctiva, and the sclera, while the vitreous and internal limiting 
membrane (ILM) will still need to be crossed. The vitreous has a gel-like network structure with a pore size expected to be in the range of $500 \mathrm{~nm}$. Small drugs, proteins, and nanoparticles may diffuse through the vitreous $[5,6]$. A negatively charge surface and size below $500 \mathrm{~nm}$ are preferred qualities for DDS because these features may improve particle mobility [6,7]. When the drug requires intracellular targeting within the retina, barriers at the level of the plasma membrane will limit it's diffusion, especially for hydrophilic molecules [8]. DDS can help the delivery of the drug [9] and nanoparticles composed with material that enhances endocytosis, such as solid lipid nanoparticles (SLP), will likely improve the entrance of the active compound into the target cells [10-12].

Small hydrophilic cargo, like DNA [13] or cGMP analogue [14], are of major interest for treatment development. However, encapsulation of small hydrophilic molecules is challenging. Multiple DDS have been previously considered, such as liposomes [15] and polymeric particles [16]. However, these systems are characterized by low encapsulation efficiency, undesired leakage, or initial burst release [17]. For instance, when the water-in oil-in water $\left(\mathrm{W}_{1} / \mathrm{O} / \mathrm{W}_{2}\right)$ emulsion method is used to prepare lipid based particles such as liposomes, a small hydrophilic molecule can suffer low encapsulation efficiency due to leakage during preparation [18]. Other lipid based particles, like SLPs, are limited in space for uploading hydrophilic molecules [19] and compounds may be expelled following the polymorphic transition of the structure during storage [20]. To accommodate larger amounts of active molecules in the core of lipid based particles, and prevent their premature release, the addition of a thermo-responsive gel core [21] or of micelles [22,23] have been reported and shown to improve the encapsulation of hydrophilic cargo, such as proteins.

In this study we developed a new DDS formulated to hold small hydrophilic molecules by (i) incorporation of the compound inside a hydrogel, which forms the core of a SLP and (ii) combining a lipid and a hydrophobic polymer in the nanoparticle shell composition to improve its physicochemical characteristics. Rhodamine B (RhoB) was selected as a model drug/small hydrophilic cargo during development and the DDS encapsulation capability was validated using a phosphorothioate analogue of cGMP, named Rp-8-Br-PET-cGMPS (CNo3), a compound that previously demonstrated to hold in vivo neuroprotective properties in the retina [24]. The resulting DDS was anionic with average particle size around $250 \mathrm{~nm}$ and high encapsulation efficiency (60\%), characteristics important to overcome constrains for intravitreal route delivery (i.e., size, surface charge, encapsulation efficiency, and internalization ability). An in vitro test using photoreceptor and retinal pigment epithelium cell lines indicated that the DDS could help the process of small hydrophilic cargo internalization.

\section{Methods}

\section{Materials}

Poloxamer 407 (Sigma Aldrich), poloxamer 188 (Applichem), rhodamine B (RhoB; Sigma Aldrich), Rp-8-Br-PET-cGMPS, also known as CNo3 (provided by Research Institute of Sweden), Glycerol tripalmitate (GTP; Alfa Aesar), soy-bean lecithin (LCT; VWR), stearic acid (SA; BASF), 50/50 DL-lactide/glycolide copolymer (PLGA; Corbion), poly- $\varepsilon^{-}$ caprolactone 14kDa (PCL; Sigma Aldrich), 8-aminonaphthalene-1,3,6-trisulfonic acid disodium salt (ANTS; Biotium), p-Xylene-Bis-Pyridinium Bromide (DPX; Biotium), dichloromethane (VWR), deionized water (VWR), ARPE-19 cell (ATCC), 661W cell (generously provided by Dr. Muayyad Al-Ubaidi, University of Oklahoma), Dulbecco's modified Eagle's medium and Ham's F12 nutrient mixture (DMEM/F12; Gibco), low glucose (1mg/ml) Dulbecco's modified Eagle's medium (DMEM; Gibco), fetal bovine serum (FBS; Gibco), glutamine (Sigma Aldrich), penicillin-streptomycin (Sigma Aldrich), Accutase ${ }^{\circledR}$ solution (Sigma-Aldrich), paraformaldehyde (Sigma Aldrich), anti-Zonula occludens-1 (ZO- 
1) antibody (Invitrogen), goat anti-rabbit secondary antibody (Life technologies), 4',6diamidino-2-phenylindole, dihydrochloride (DAPI; Sigma Aldrich), colorimetric methylthiazolyl diphenyl-tetrazolium bromide (Sigma Aldrich). All purchased materials were used as received.

\section{Synthesis of solid lipid nanoparticles (SLPs)}

SLPs consisting of a gel core and a composite shell have been developed using the double emulsion method with modifications [21-23]. Different formulations were prepared based on the composite shell composition (i.e., presence and type of hydrophobic polymer) as well as its core composition (i.e., presence of hydrogel core). RhoB was selected to track the particles inside the cells during the initial development. Both RhoB $(479.02 \mathrm{~g} / \mathrm{mol})$ and the CNo3 $(562.3 \mathrm{~g} / \mathrm{mol})$ are highly hydrophilic and similar in size.

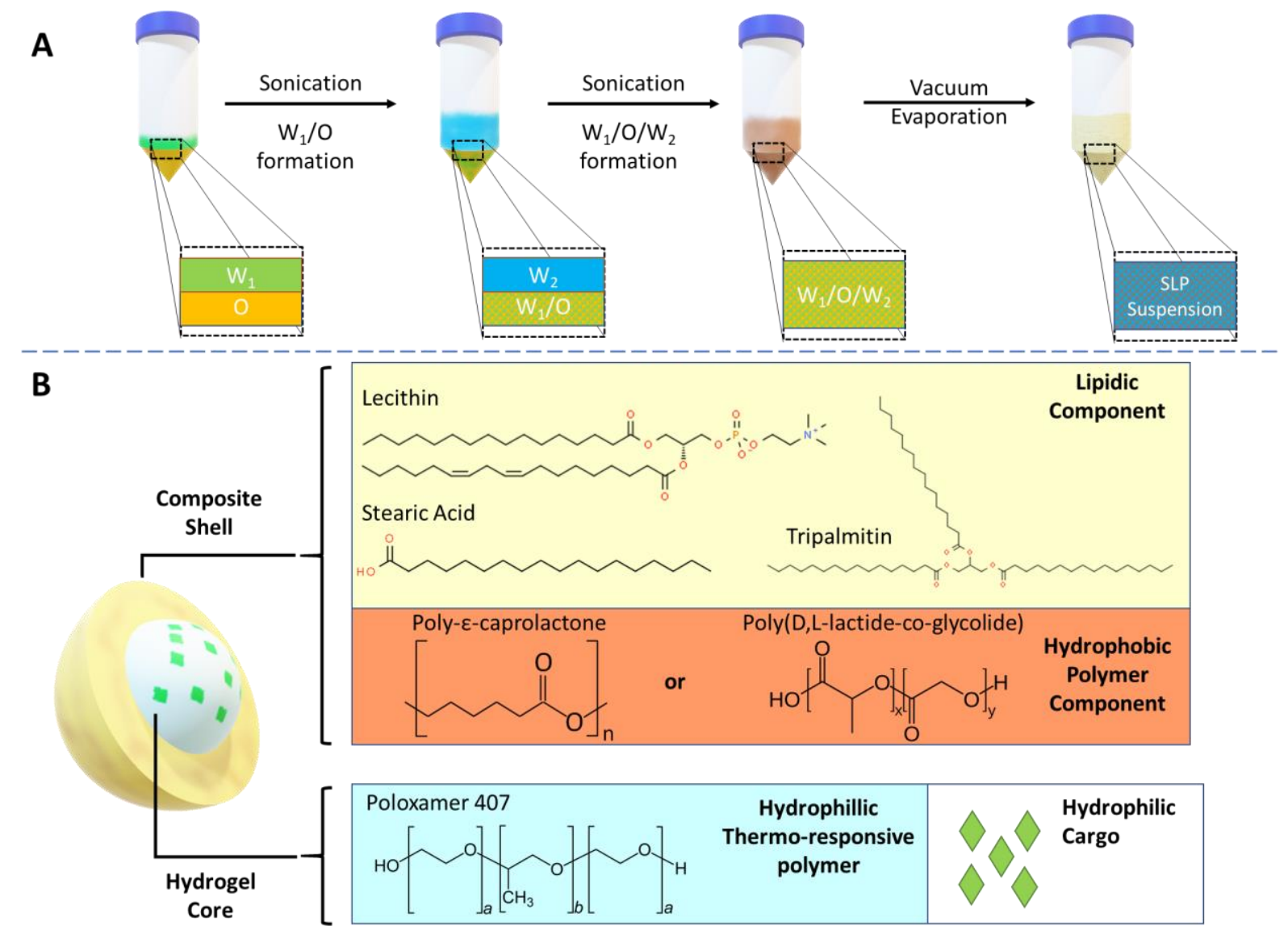

Figure 1. One pot synthesis of solid lipid nanoparticle consisting of a composite shell and a nanogel core. (A) formation of the SLPs suspension. (B) Composition of the composite SLPs.

The SLP formulation is illustrated in Figure 1. A stock solution for $\mathrm{W}_{1}$-phase without gel core was prepared by dissolving RhoB in deionized water to reach $10 \mathrm{mg} / \mathrm{ml}$ concentration. For $\mathrm{W}_{1}$-phase stock with gel core, poloxamer 407 was added to the RhoB solution to reach $40 \% \mathrm{w} / \mathrm{v}$. To ensure complete dissolution, the poloxamer 407 solution was dissolved at $4{ }^{\circ} \mathrm{C}$ for $48 \mathrm{~h}$. The $\mathrm{W}_{2}$-phase stock solution was prepared by dissolving poloxamer 188 in deionized water to reach $2 \% \mathrm{w} / \mathrm{v}$ concentration. The O-phase solution was prepared by dissolving GTP, LCT, SA, and PCL or PLGA in $1 \mathrm{ml}$ of dichloromethane according to different formulation codes listed in Table 1. Finally, for the preparation of blank particle and drug-loaded particle, deionized water and $\mathrm{CNo} 3$ were used respectively in the $\mathrm{W}_{1}$ phase instead of RhoB. Similarly, for release assays of the compound from SLPs inside the cell, ANTS and DPX were co-encapsulated in the $\mathrm{W}_{1}$ phase instead of RhoB. 
Table 1. Formulation code and component mass dissolved in O-phase for each formulation. DDS: drug delivery system; SLP: solid lipid nanoparticle; GTP: tripalmitin; LCT: soy-bean lecithin; SA: stearic acid; PCL: poly-e-caprolactone; PLGA: 50/50 DL-lactide/glycolide.

\begin{tabular}{ccccccc}
\hline DDS & GTP $(\mathrm{mg})$ & LCT $(\mathrm{mg})$ & SA $(\mathrm{mg})$ & PCL $(\mathrm{mg})$ & PLGA (mg) & Core \\
\hline SLP.01 & 15 & 15 & 1 & - & - & Aqueous \\
SLP.02 & 15 & 15 & 1 & 10 & - & Aqueous \\
SLP.03 & 15 & 15 & 1 & - & 10 & Aqueous \\
SLP.04 & 15 & 15 & 1 & - & - & Hydrogel \\
SLP.05 & 15 & 15 & 1 & 10 & - & Hydrogel \\
SLP.06 & 15 & 15 & 1 & - & 10 & Hydrogel \\
\hline
\end{tabular}

The synthesis was performed by adding $200 \mu \mathrm{l}$ of $\mathrm{W}_{1}$-phase stock solution kept at $4-7^{\circ} \mathrm{C}$, using an ice bath, to $1 \mathrm{ml}$ of O-phase solution followed by sonication with the Q55 ultrasound probe from Qsonica (amplitude: 30\% for 60 $\mathrm{s}$ without a pulse) to form the primary $\mathrm{W}_{1} / \mathrm{O}$ emulsion. Then, $4.8 \mathrm{ml}$ of $\mathrm{W}_{2}$-phase solution was added into the primary emulsion and sonicated to form $\mathrm{W}_{1} / \mathrm{O} / \mathrm{W}_{2}$ emulsion (amplitude: $40 \%$ for $10 \mathrm{~s}$ followed by $20 \mathrm{~s}$ at $20 \%$ amplitude without a pulse). The solution was further diluted with $10 \mathrm{ml}$ of $W_{2^{-}}$ phase solution followed by sonication (amplitude: $20 \%$ for $30 \mathrm{~s}$ without a pulse). The organic solvent was then removed by vacuum evaporation at room temperature for 20 minutes to form the nanoparticles. The resulting colloidal solution was stirred for $4 \mathrm{~h}$ to ensure complete removal of dichloromethane.

For the analyses of compound release inside the cells, ANTS $(25 \mu \mathrm{M})$ and DPX (90 $\mu \mathrm{M})$ as a pair of tracer and quencher were co-encapsulated in the SLPs that were analyzed by a fluorescence quenching assay [25].

\section{Physicochemical characterization}

Dynamic laser scattering. The particle size distribution and hydrodynamic diameter were measured using NanoPhox DLS equipment from Sympatec, GmbH. Before the measurement, concentrated particle solution was diluted 5 times using $2 \% \mathrm{w} / \mathrm{v}$ poloxamer 188 solution. The analysis was performed using a non-negative least square (NNLS) algorithm integrated in Windox5 software from Sympatec, GmbH. Viscosity of the solution was calibrated and validated using the polystyrene bead standard. For each sample, measurement was repeated 6 times and each lasted 200 seconds.

Zeta potential. The zeta potential of the particle was measured by Zetasizer equipment from Malvern. Samples were diluted 5 times using $10 \mathrm{mM}$ phosphate buffer, $\mathrm{pH}$ 7.4. For each sample, measurement was repeated 3 times.

Morphological analysis. SLP solution as described in table 1 was synthesized without hydrophilic cargo (i.e., blank particle) and analyzed using a transmission electron microscope (TEM). The colloidal nanoparticle solution was stained with $7 \%(\mathrm{w} / \mathrm{v})$ phosphotungstic acid as a negative contrast. The morphological characterization of the particle was performed at an TEM acceleration voltage of $120 \mathrm{kV}$.

Encapsulation Efficiency. Encapsulation efficiency (EE) was measured using an indirect method in which the amount of unencapsulated cargo outside the particles was measured. Sample solutions were filtered using a $100 \mathrm{kDa}$ microcentrifuge membrane filter (Sartorius) ( $3 \times 5$ minutes, @500o RCF). The filtrate was collected, and the amount of cargo was quantified using equation 1. As there might be some loss of W1-Phase in the pipette tips during synthesis, cargo loss was quantified to avoid encapsulation efficiency overestimation. 


$$
E E(\%)=\left(1-\frac{\text { cargo amount detected in filtrate }}{\text { Theoretical cargo amount }- \text { cargo loss in tips }}\right) \times 100 \%
$$

RhoB and $\mathrm{CNO} 3$ concentrations were quantified by measuring absorbance at $550 \mathrm{~nm}$ and at $254 \mathrm{~nm}$ using an UV-spectrophotometer (Biomolecular device) and high-pressure liquid chromatography (HPLC) system (Dionex Ultimate 3000), respectively. For HPLC, mobile phases A and B were $5 \mathrm{mM}$ ammonium acetate buffer, and acetonitrile, respectively. $5 \mu \mathrm{l}$ of the sample were injected into the column (Waters ${ }^{\circledR}$ XBridge C18 XP column, $50 \times 3$ $\mathrm{mm}$ ). The HPLC quantification was performed using Chromeleon software by calculating the peak area in the retention time around 3.1 minutes. Experiment was replicated 9 times for UV-spectroscopy and 3 times for HPLC.

\section{In Vitro cell culture}

The human retinal pigment epithelial cell line (ARPE-19), was cultured in Dulbecco's modified Eagle's medium and Ham's F12 nutrient mixture (DMEM/F12) supplemented with $10 \%$ foetal bovine serum (FBS) and penicillin-streptomycin in a $5 \% \mathrm{CO}_{2}$ incubator at $37^{\circ} \mathrm{C}$. The $661 \mathrm{~W}$ cell line was cultured in low glucose $(1 \mathrm{mg} / \mathrm{ml})$ Dulbecco's modified Eagle's medium (DMEM) supplemented with $10 \%$ FBS, $2 \mathrm{mM}$ glutamine and penicillinstreptomycin in a $5 \% \mathrm{CO}_{2}$ incubator at $37^{\circ} \mathrm{C}$. Approximately every three days cell cultures reached $70-80 \%$ confluence and the cells were passaged and sub-cultivated.

\section{Fluorescence microscopic analysis and Immunofluorescence}

Cells were seeded on glass coverslips in a 24-well plate at a density of $4 \times 10^{4}$ cells/well. $24 \mathrm{~h}$ later, cells were treated with RhoB encapsulated nanoparticles solution. As control, cells were treated with free RhoB solution at the same concentration $(20 \mu \mathrm{M})$ of RhoB present in the nanoparticle solution. A blank was prepared by incubating the cells with nanoparticles containing no fluorophores. For compound release assays, $24 \mathrm{~h}$ after seeding, ARPE-19 cells were treated for 5 hours with medium containing $200 \mu \mathrm{g} / \mathrm{ml}$ blank SLP (blank), freely dissolved tracer or tracer/quencher pair (control), or $200 \mu \mathrm{g} / \mathrm{ml}$ SLP loaded with ANTS/DPX. Afterwards, the medium was replaced with non-treated fresh medium and the incubation was protracted for $24 \mathrm{~h}, 48 \mathrm{~h}$ or $72 \mathrm{~h}$.

After incubation, cells were rinsed with phosphate buffer saline (PBS), fixed with 2\% paraformaldehyde (PFA) for 10 minutes and nuclei were stained with $0.1 \mu \mathrm{g} / \mathrm{ml} \mathrm{4^{ \prime } , 6 -}$ diamidino-2-phenylindole (DAPI). For the immunofluorescence, after fixing with 2\% PFA for 10 minutes, cells were incubated with anti-ZO1 (1:100) primary antibody overnight at $4^{\circ} \mathrm{C}$. After washing three times with PBS, cells were incubated with the Alexa Fluor ${ }^{\circledR} 488$ goat anti-rabbit secondary antibody (1:1000) and $0.1 \mu \mathrm{g} / \mathrm{ml}$ DAPI for $40 \mathrm{~min}$ at room temperature. Slides were mounted with Mowiol 4-88 and cells were observed using the Zeiss Axio Imager A2 fluorescence microscope.

\section{Cell viability assay}

Cell viability assay was performed by colorimetric methyl-thiazolyl diphenyltetrazolium bromide (MTT) assay. Cells were cultured in a 96-well plate at a density of 6,000 cells/well. After treatment with SLPs for different times, the medium was aspirated, and cells were incubated with $50 \mu \mathrm{l}$ of $1 \mathrm{mg} / \mathrm{ml} \mathrm{MTT}$ solution for 90 minutes at $37^{\circ} \mathrm{C}$. The supernatant was removed, and the purple formazan crystals were dissolved in $100 \mu$ isopropanol. The plate was shaken for 10 minutes and analysed at $570 \mathrm{~nm}$ using a microplate reader (Labsystems Multiskan MCC/340). The average absorbance was obtained from 3 biological replicates.

\section{Flow cytometry analysis}


ARPE-19 and 661W cells were seeded on 12-well plates at a density of $1 \times 10^{5}$ cells/well. After treatment with control or SLPs, cells were detached with $500 \mu$ Accutase ${ }^{\circledR}$ and collected by centrifugation at $300 \mathrm{G}$ for 5 minutes at room temperature. The cells were washed three times with $500 \mu \mathrm{l}$ PBS and collected by centrifugation at 300xg for 5 minutes at room temperature. The cell pellet was resuspended with $500 \mu$ l of PBS and RhoB fluorescence was immediately analyzed at the Attune ${ }^{\circledR}$ NxT Acoustic Focusing Cytometer. The channel voltage and gain were maintained constant throughout the whole analysis.

Statistical analysis.

Statistical analysis was performed using ANOVA, and $\mathrm{p}<0.05$ was considered significant.

\section{Results}

\section{DDS development and analytical study.}

With the purpose of developing a DDS to facilitate the uptake of hydrophilic molecules in retinal cells, we developed a procedure for SLPs with a size range of 200-250 $\mathrm{nm}$ (Table 2). The addition of hydrophobic polyesters such as PCL and PLGA notably improved particle polydispersity index to less than 0.4 , and all nanoparticles produced were anionic as characterized by their zeta potential. The surface charge was similar for particles with and without hydrogel, e.g., SLP.03 $(-27 \pm 2.3 \mathrm{mV})$ versus SLP.06 $(-24 \pm 1.5 \mathrm{mV})$. On the other hand, the presence of a hydrogel core improved the encapsulation efficiency, e.g., SLP.02 $(24 \pm 0.8 \%)$ versus SLP.05 (47 $\pm 1.4 \%)$. Compared to SLP.01 (19 $\pm 1.7 \%)$, adding hydrophobic polyester to the shell formulation had no detectable effect on encapsulation efficiency, i.e., all particles with an aqueous core had a RhoB encapsulation efficiency around $20 \%$, while those with a hydrogel core could encapsulate above $40 \%$ of cargo regardless of the shell type. The DDS morphology observed using TEM confirmed that measured particles were below $500 \mathrm{~nm}$ as required for mobility in the vitreous (Figure 2).

Table 2. Summary of DDS physicochemical characteristic. SLP: solid lipid nanoparticle; PCL: poly- $\varepsilon$-caprolactone; PLGA: 50/50 DL-lactide/glycolide; PDI: polydispersity index; EE: Encapsulation Efficiency.

\begin{tabular}{lccccccc}
\hline DDS & Shell & Core & Cargo & $\begin{array}{c}\text { Size } \\
(\mathrm{nm})\end{array}$ & PDI & $\begin{array}{c}\text { Zeta } \\
\text { Potential } \\
(\mathrm{mV})\end{array}$ & EE (\%) \\
\hline SLP.01 & Lipid & Aqueous & RhoB & $218 \pm 16.0$ & $0.44 \pm 0.02$ & $-39 \pm 2.1$ & $19 \pm 1.7$ \\
SLP.02 & Lipid, PCL & Aqueous & RhoB & $239 \pm 3.2$ & $0.30 \pm 0.03$ & $-15 \pm 1.6$ & $24 \pm 0.8$ \\
SLP.03 & Lipid, PLGA & Aqueous & RhoB & $228 \pm 0.9$ & $0.34 \pm 0.04$ & $-27 \pm 2.3$ & $19 \pm 2.8$ \\
SLP.04 & Lipid & Hydrogel & RhoB & $252 \pm 19.3$ & $0.44 \pm 0.07$ & $-41 \pm 1.2$ & $44 \pm 3.0$ \\
SLP.05 & Lipid, PCL & Hydrogel & RhoB & $225 \pm 2.3$ & $0.22 \pm 0.04$ & $-13 \pm 1.6$ & $47 \pm 1.4$ \\
SLP.06 & Lipid, PLGA & Hydrogel & RhoB & $237 \pm 9.4$ & $0.14 \pm 0.05$ & $-24 \pm 1.5$ & $45 \pm 2.6$ \\
SLP.05 & Lipid, PCL & Hydrogel & CNO3 & $257 \pm 4.1$ & $0.33 \pm 0.03$ & $-21 \pm 3.9$ & $60 \pm 0.9$ \\
SLP.06 & Lipid, PLGA & Hydrogel & CNO3 & $229 \pm 3.6$ & $0.38 \pm 0.01$ & $-25 \pm 3.0$ & $59 \pm 1.0$ \\
\hline
\end{tabular}




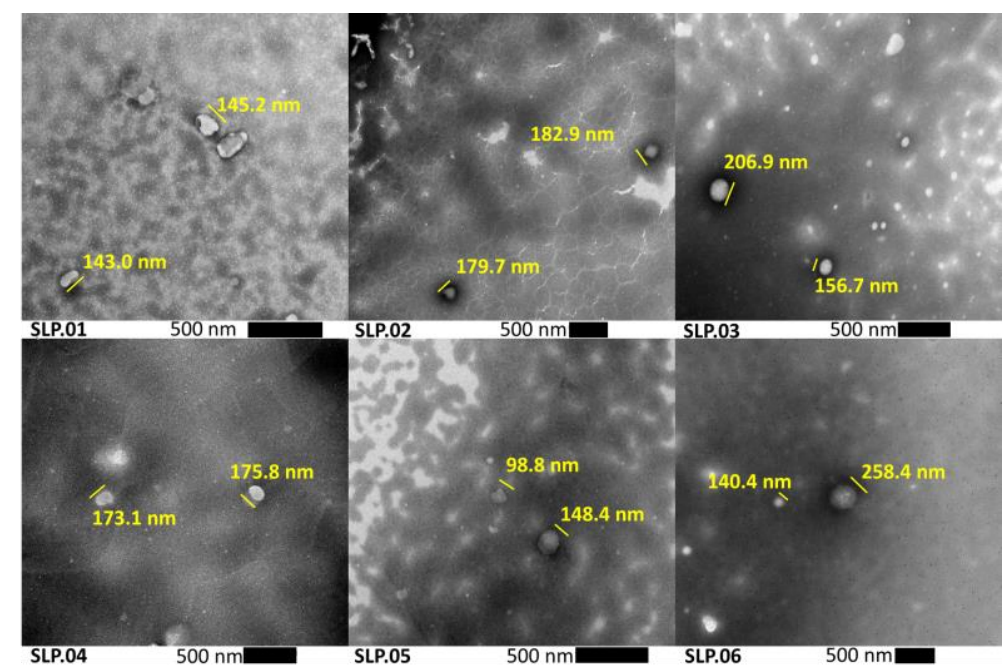

Figure 2. TEM analysis of particle morphology. Blank SLP particle without cargo was used for analysis.

Based on the analytical analysis of the different SLP generated, we chose to focus on SLP.05 and SLP.06 because they showed the best encapsulation efficiency together with a size below $500 \mathrm{~nm}$ and a negative surface charge, features necessary for intravitreal drug delivery to retina. Small size and negative charge should help the SLP to travel more freely within the vitreous $[6,7]$.

\section{In vitro cytotoxicity of nanoparticles}

We then wanted to assess if these SLP could be taken up by retinal cells. In fact, active molecules should be internalized to reach their targets in RPE and photoreceptors, the cells primarily affected in retinal degeneration. For this study we chose two retinal cell types: ARPE-19, a spontaneously arising human RPE cell line with normal karyotype [26]; and $661 \mathrm{~W}$ photoreceptor-like cells derived from a mouse retinal tumor generated in a transgenic mouse expressing SV40 large T-antigen under the control of IRBP (interphotoreceptor retinoid-binding protein) promoter [27].

We first wanted to assess whether and at which concentration the newly developed SLPs might be toxic to retinal cells, an important information for the prospective used in the clinic. To this purpose, a cell viability assay was performed on ARPE-19 and 661W cells. Cells were exposed to scaling concentrations of SLP.05 and SLP.06 nanoparticles for different time points.
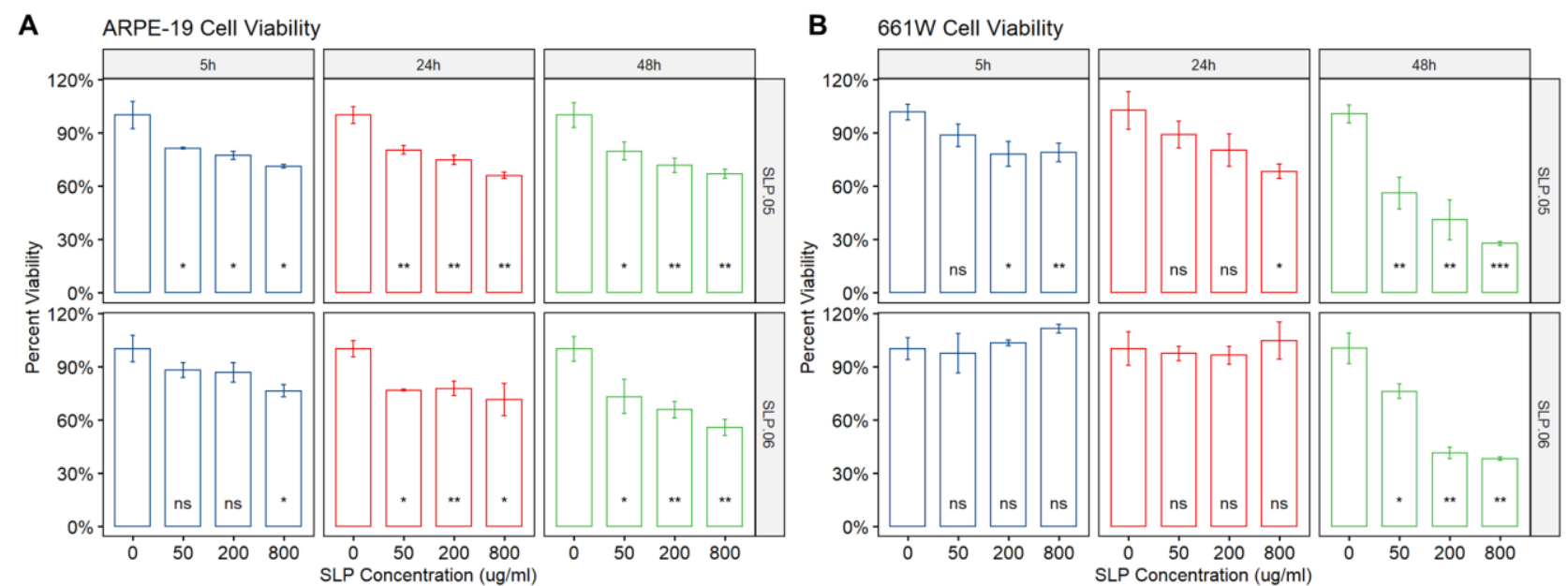

Figure 3. Cell viability study of ARPE-19 and 661W cells exposed to SLP.05 and SLP.06 at increasing concentrations (from o to $800 \mu \mathrm{g} / \mathrm{ml}$ ) and for different duration time. Pairwise comparison against control at different time points for (A) ARPE19 and (B) 661W cell line. ${ }^{*} \mathrm{P}<0.05,{ }^{* *} \mathrm{P}<0.01$, ns: not significantly different, $\mathrm{n}=3$. 
SLP.05 and SLP.06 displayed a dose-dependent toxicity on ARPE-19 cells (Figure 3A). A higher tolerance to SLPs was observed on $661 \mathrm{~W}$ cells compared to ARPE-19, especially at $5 \mathrm{~h}$ and $24 \mathrm{~h}$ exposure time but toxicity was observed after prolonged exposure (Figure $3 \mathrm{~B}$ ). SLP.05 had a dose-dependent toxicity, which was maintained also at longer times of exposure and a drop in cell viability after $24 \mathrm{~h}$ of exposure was observed at all the tested concentrations. SLP.o6, at concentrations up to $800 \mu \mathrm{g} / \mathrm{ml}$, were quite well tolerated by $661 \mathrm{~W}$ cells in the first $24 \mathrm{~h}$ of exposure.

\section{Nanoparticle uptake by $661 \mathrm{~W}$ and ARPE-19 cells}

To define if the nanoparticles could be uptaken by the cells, SLP.05 and SLP.06 loaded with RhoB were applied to the $661 \mathrm{~W}$ cells at increasing concentrations (Figure 4) for $5 \mathrm{~h}$ a time of exposure with limited toxicity. We observed an increasing fluorescence signal in correlation to the concentrations of SLP.05 (Figure 4A) and SLP.06 inside the cells (Figure 4B). RhoB loaded SLP.05 and SLP.06 were localized in the cytosol and excluded from the nuclei (blue) indicating internalization but not nuclear transfer.

When $200 \mu \mathrm{g} / \mathrm{ml}$ RhoB loaded SLP.05 and SLP.06 were applied to the ARPE-19 cells for $5 \mathrm{~h}$, both types of SLPs were uptaken when compared to free RhoB or un-loaded SLPs (Figure 5). By labelling the cell boundaries with an antibody anti-zonula occludens-1 (ZO-1), we confirmed that RhoB loaded SLPs localized in the cytosol and did not enter the nucleus (Figure 6).

A
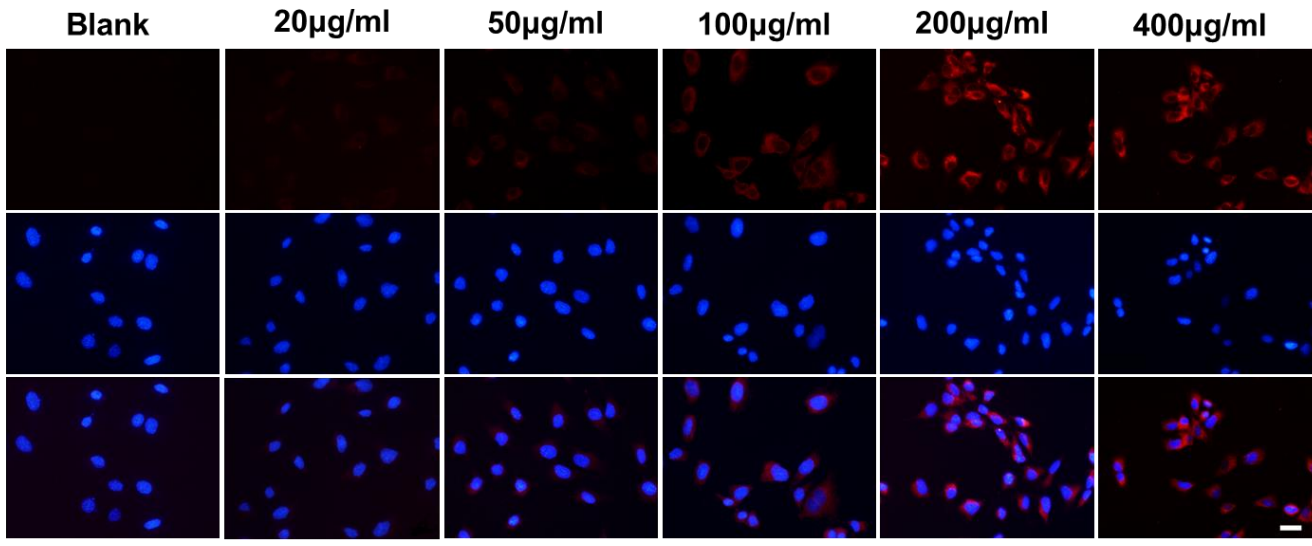

B

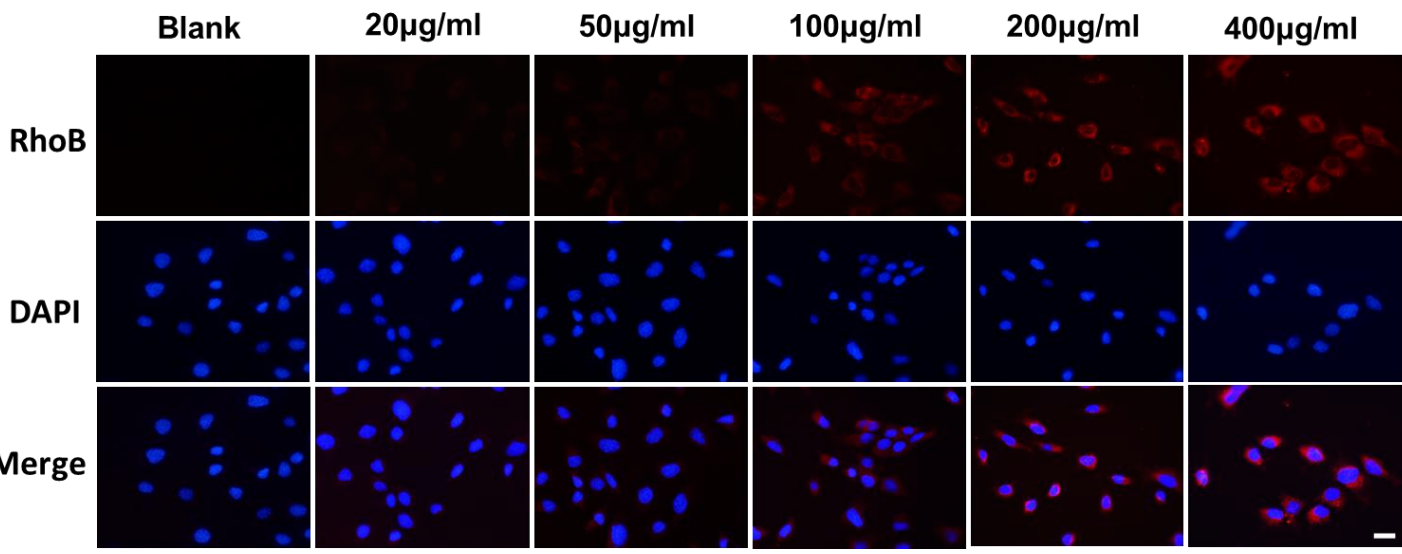

Figure 4. Fluorescence analysis of 661W cells following treatments with RhoB (red) loaded SLPo.5 (A) and SLP.o6 (B) for $5 \mathrm{~h}$ at different concentrations. Blank: Cells treated with $200 \mu \mathrm{g} / \mathrm{ml} \mathrm{SLP.05}$ or SLP.06 without loading dye. Nuclei were stained with DAPI. RhoB concentration was kept constant at $20 \mu \mathrm{M}$ for different SLP concentration. Scale bars: $20 \mu \mathrm{m}$. 


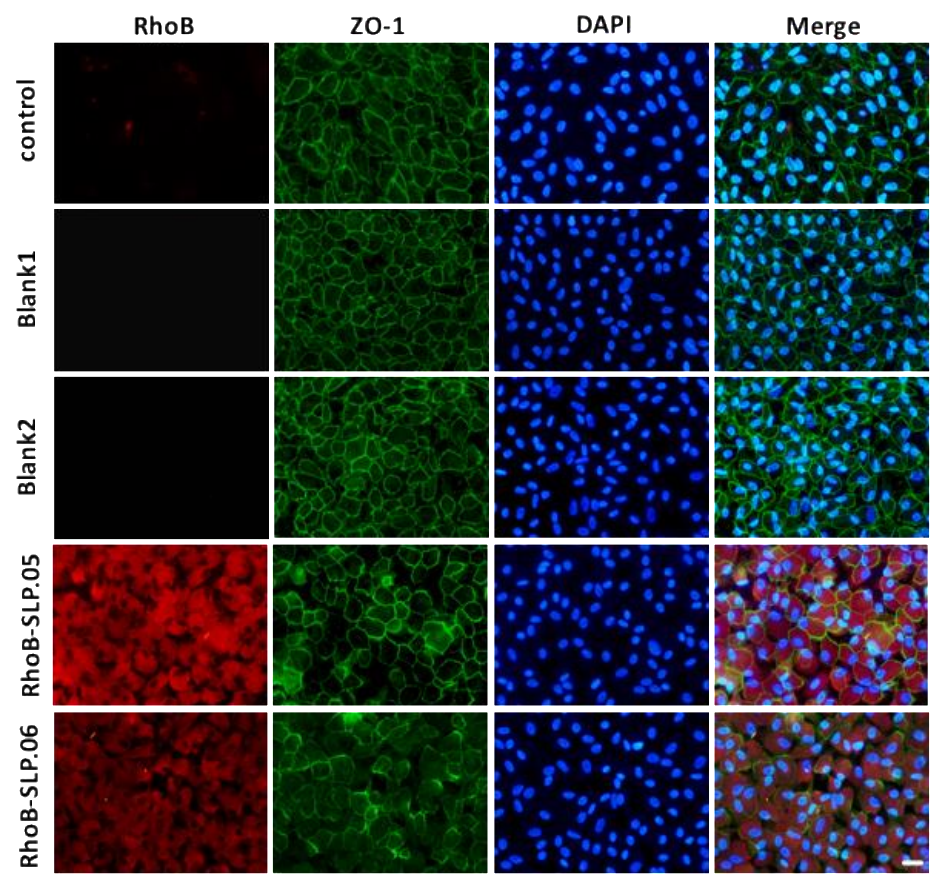

Figure 5. Immunofluorescent analyses of ARPE-19 cells following incubation for $5 \mathrm{~h}$ with $200 \mu \mathrm{g} / \mathrm{ml}$ RhoB (red) loaded SLP.05 and SLP.o6. Control: cells cultured with freely dissolved $20 \mu \mathrm{M}$ RhoB dye; Blank1: cells cultured with $200 \mu \mathrm{g} / \mathrm{ml}$ SLP.05 without dye; Blank2: cells cultured with $200 \mu \mathrm{g} / \mathrm{ml} \mathrm{SLP.06} \mathrm{without} \mathrm{dye.} \mathrm{RhoB} \mathrm{concentration} \mathrm{in} \mathrm{RhoB} \mathrm{loaded} \mathrm{SLP}$ is the same as in the control $(20 \mu \mathrm{M})$. Tight junctions marking the boundaries of the cells were labelled using an antibody anti-ZO-1 (green). Nuclei were stained with DAPI. Scale bar: $20 \mu \mathrm{m}$.

To partially characterize the mechanism of SLP uptake, 661w cells were cultured with RhoB loaded SLP.o5 and SLP.o6 for $1 \mathrm{~h}$ at either $37^{\circ} \mathrm{C}$ or $4^{\circ} \mathrm{C}$. Cells incubated at $4^{\circ} \mathrm{C}$ could not efficiently uptake SNPs, based on the absence of fluorescent signal, suggesting that energydependent endocytosis possibly mediated SLPs uptake in the cells [28].

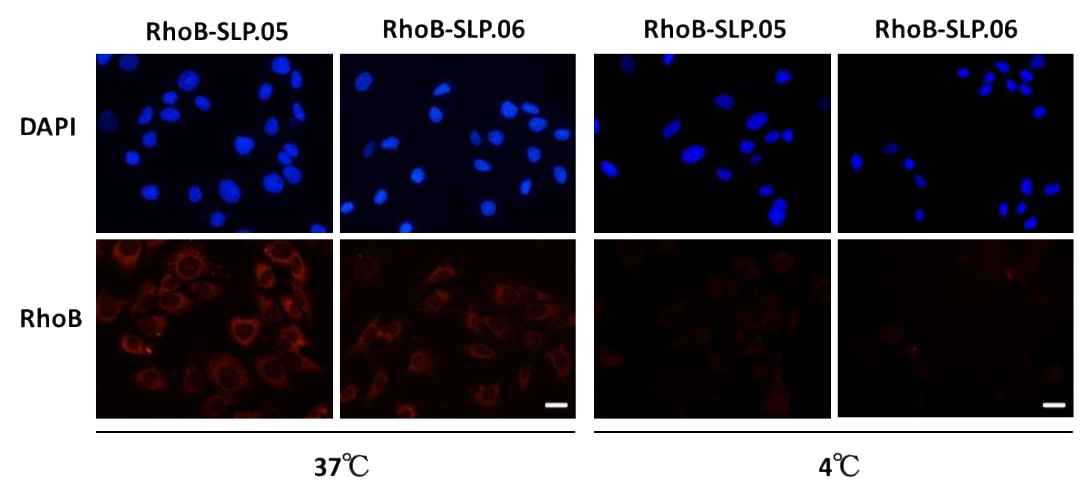

Figure 6. Fluorescence analysis of $661 \mathrm{~W}$ cells following incubation for $1 \mathrm{~h}$ with $200 \mu \mathrm{g} / \mathrm{ml}$ RhoB loaded SLP.05 and SLP.06 at either $37^{\circ} \mathrm{C}$ or $4^{\circ} \mathrm{C}$; RhoB concentration: $20 \mu \mathrm{M}$; Scale bars: $20 \mu \mathrm{m}$.

\section{Quantification of nanoparticle internalization}

A time course flow cytometry analysis was performed to quantify the uptake of SLP in the two retinal cell types. ARPE-19 and $661 \mathrm{~W}$ cells were treated with $200 \mu \mathrm{g} / \mathrm{ml}$ of RhoBloaded SLP.05 and SLP.06, and the fluorescence signal was quantified at different incubation times ( $1 \mathrm{~h}, 3 \mathrm{~h}, 5 \mathrm{~h}$, and $24 \mathrm{~h}$ ) by flow cytometry. Non-treated ARPE-19 and 661W cells were used as control. Gating was performed twice, with R1 gate to select the cell population and R2 gate to select RhoB positive cells based on blue laser (BL2-A) detector as shown in Figure 7A.

The graphical summary of the result is plotted over time in Figure $7 \mathrm{~B}$. The amount and intensity of fluorescent cells increased with prolonged incubation times. Notably, after $3 \mathrm{~h}$ 
of incubation, around $48 \%$ of ARPE-19 cells had internalized SLP.05 particles and internalization increased to over $67 \%$ in $5 \mathrm{~h}$. Uptake of SLP.06 was less efficient and required $24 \mathrm{~h}$ to reach about $16 \%$ (Figure $7 \mathrm{~B}$ ). $661 \mathrm{~W}$ cells showed less internalization compared to ARPE-19 cells, with $13 \%$ RhoB on SLP.05 and $15 \%$ on SLP.06 after $5 \mathrm{~h}$ of exposure. In general, ARPE-19 cells displayed an increased SLP internalization with SLP.05 compared to 661W. On the other hand, SLP.06 showed similar SLP internalization levels between the two cell types.
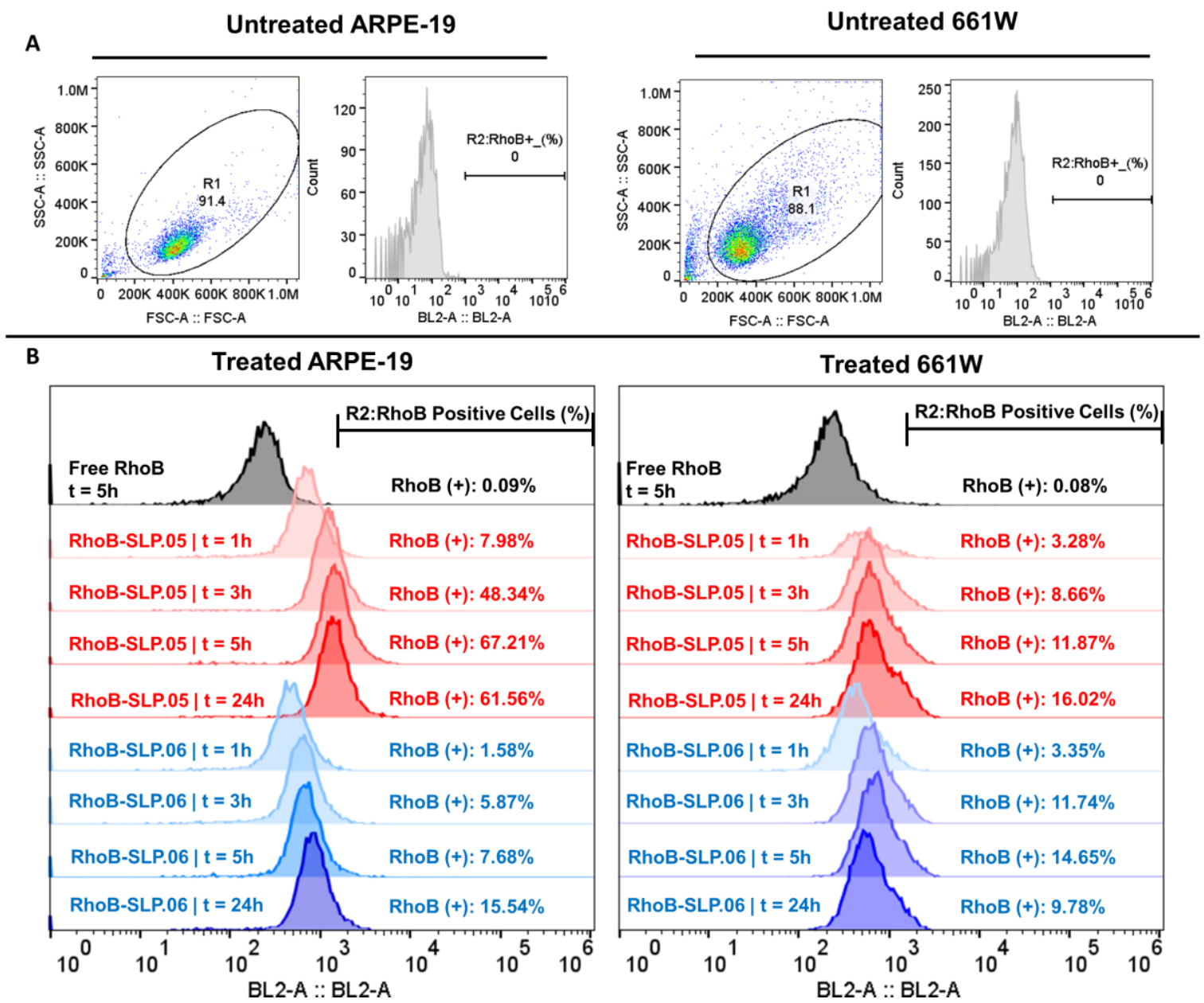

Figure 7. Flow cytometry analysis of fluorescence signal in ARPE-19 and 661W cells incubated with 200 $\mu \mathrm{g} / \mathrm{ml}$ RhoB loaded SLP.05 and SLP.06 at different time points. (A) Gating controls on each cell line for determining RhoB positive cells. (B) Histogram overlay of RhoB relative fluorescence intensity and RhoB positive cell percentage over different incubation time points. RhoB (+): percentage of cells with a fluorescent signal above the threshold defined in panel A. R1: Gating for selecting cells population; R2: Gating to select RhoB positive cells based on blue laser (BL2-A) detector; FSC-A: Forward scattering channel; SSC-A: Side scattering channel.

\section{Release of the encapsulated cargo inside the cells}

After demonstration of SLP internalization in retinal cells we wondered if the nanoparticle could release the encapsulated cargo after uptake. To address this issue, we coencapsulated ANTS/DPX, a system previously published for fluorescence quenching assays to study vesicle leakage [29]. ANTS is a fluorescent dye that is quenched upon collision to the DPX molecule. ANTS fluorescence will increase upon breakage of SLP shell and release of cargo, because the quenching by DPX will diminish (i.e., increase of molecular distance leads to low collision probability) (Figure 8A). Based on this assay we could confirm that SLP could release the encapsulated compound after endocytosis. We observed that, overtime, a fluorescence signal could be detected in ARPE-19 cells exposed to SLP.05 and SLP.o6 co-encapsulated with ANTS/DPX. The fluorescent signal was especially evident after 
$48 \mathrm{~h}$. These data confirmed that the encapsulated cargo could be released from the nanoparticles once they entered cells.

Based on these promising results, as a proof of principle we evaluated if the newly developed SLPs could load a previously published compound, CNO3, demonstrated to be neuroprotective for the retina [24]. We were able to achieve about $60 \%$ encapsulation efficiency of $\mathrm{CNO} 3$ while maintaining a negative charge and low size in SLP.05 and SLP.06 types of nanoparticles (Table 2).

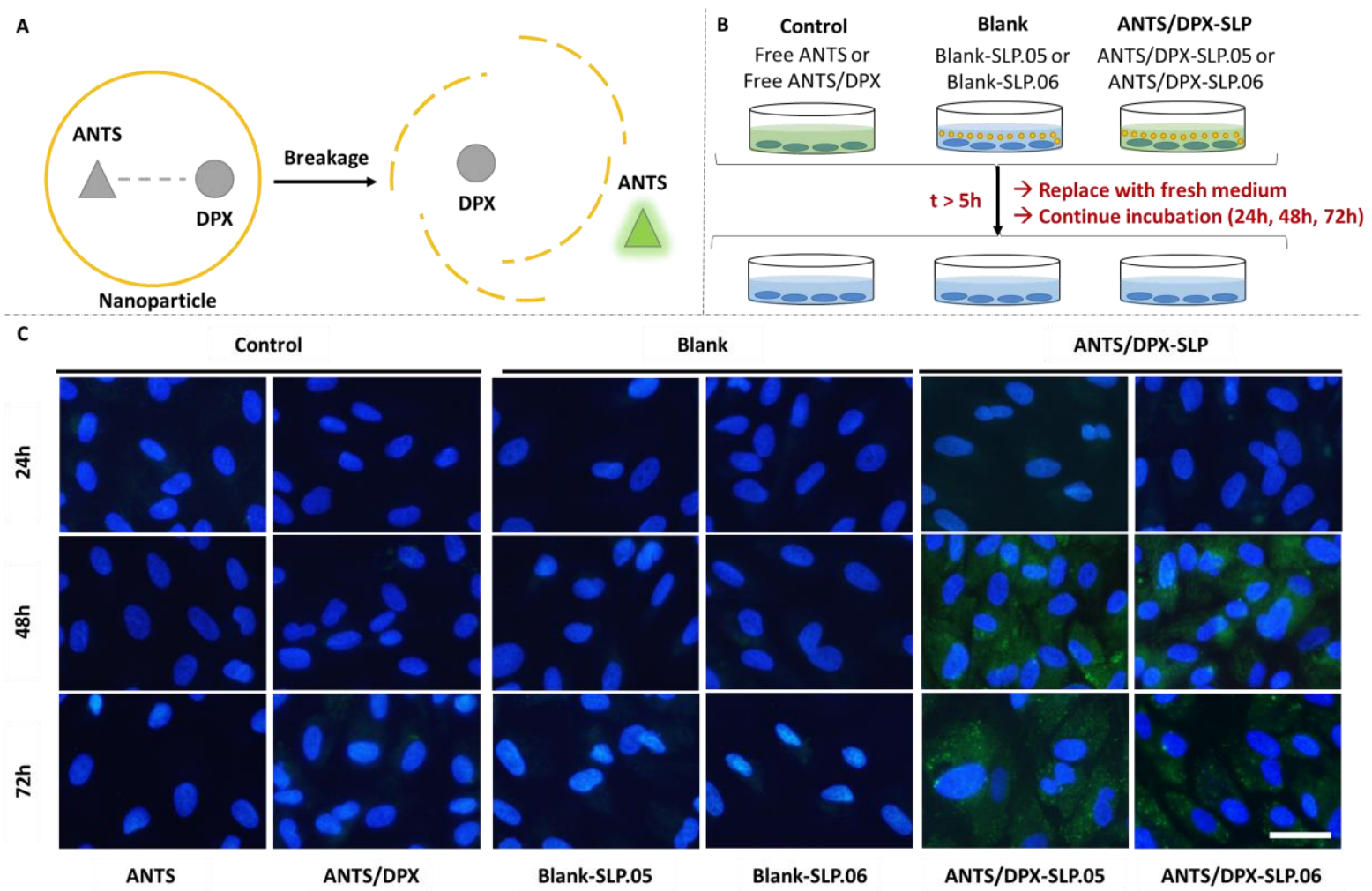

Figure 8. SLP breakage and its subsequent cargo release over time inside the cell. (A) Schematic representation of the concept of ANTS (fluorescence tracer) and DPX (quencher) usage. (B) Experimental setup: cells were incubated with control, blank and ANTS/DPX loaded SLPs for $5 \mathrm{~h}$, then the medium was replaced with fresh medium and incubation continued for $24 \mathrm{~h}, 48 \mathrm{~h}$ and $72 \mathrm{~h}$. (C) Fluorescence microscopy images taken at different incubation time points after $5 \mathrm{~h}$ incubation with ANTS/DPX loaded SLPs. Control: cells cultured with ANTS or ANTS/DPX; Blank: cells cultured with $200 \mu \mathrm{g} / \mathrm{ml} \mathrm{SLP.05}$ or SLP.06 without any dye. ANTS/DPX-SLP: cells cultured with ANTS/DPX loaded SLP.05 or SLP.06. SLP concentration: $200 \mu \mathrm{g} / \mathrm{ml}$; ANTS concentration: $25 \mu \mathrm{M}$; DPX concentration: $90 \mu \mathrm{M}$ for. Scale bar: $20 \mu \mathrm{m}$.

\section{Discussion}

The delivery of a drug to the neural retina is challenging due to the different barriers that need to be crossed and the physicochemical environment of the vitreous that needs to be considered. In this study we presented the formulation of nanoparticles which can enter retinal cells while having features that may facilitate the navigation across the vitreous. In the generation of the new SLP the key findings were: (i) the hydrogel core improved the encapsulation efficiency by up to 2-fold; (ii) the addition of hydrophobic polymer to the shell reduced particle polydispersity and improved the particle morphology. Overall, the SLPs generated here complied with the physicochemical property requirements for effective drug delivery to the neural retina by intravitreal route. Our results on encapsulation efficiencies suggested that the hydrogel core could improve the hydrophilic cargo retention during formulation. Most likely, the improved retention comes from the solidification of Poloxamer 407 emulsion droplets. This is in agreement with previously reported results showing that, thanks to the local increase in temperature during the sonication process, poloxamer 407 droplets formed a nanogel [21]. Meanwhile, the addition of polyester to the shell formulation reduced the strong negative charge of purely lipid SLPs shell (SLP.01; -39 mV) to different 
extents depending on the hydrophobic polymer used. Particle shells containing PCL (SLP.02; -15 mV) had a higher zeta potential reduction compared to PLGA (SLP.03; -27 mV). Thus, the choice of polymeric components in the SLP composite shell may be used to tailor specific surface charge.

The size of the SLP was maintained in the range of $200-250 \mathrm{~nm}$. There was an increase in surface charge when CNo3 salts were used instead of RhoB in the SLP shell that contained PCL (SLP.05). Yet, this was not observed with the shell containing PLGA (SLP.o6). Without CNo3 salts, SLP.05 $(-13 \mathrm{mV})$ had a lower charge than SLP.06 (-24 mV). This finding indicates that unencapsulated $\mathrm{CNO} 3$ salts could preferably adsorb more on the less negatively charged surface. We also observed an increase in polydispersity when CNo3 salts were used as cargo. Finally, there was about $15 \%$ increase in encapsulation efficiency of CNo3 compared to RhoB. This may be due to the almost complete insolubility of CNo3 salt in dichloromethane compared to RhoB. $\mathrm{CNo}_{3}$, thus, hardly leaks out from $\mathrm{W}_{1}$ phase during DDS preparation. In term of adaptability, we surmise that this system may also work for other small hydrophilic cargos with lower solubility in the organic solvent (e.g., DNA).

Internalization studies in two retinal cell types demonstrated that: (i) the DDS formulation helped internalization of small hydrophilic compounds; (ii) the DDS shell component might be used to tailor the uptake rate in different cell types. We observed that ARPE-19 cells could better uptake SLP containing PCL in the shell (SLP.05). This might be due to the fact that SLP.05 is less negatively charged compared to SLP.06 and the uptake level is directly affected by physicochemical properties of SLPs, such as shape, size and surface charge [30]. In 661W cells, the uptake profile of SLP.06 nanoparticle was similar as for APRE-19 cells and limited to a low percentage of cells that internalized the nanoparticles. For SLP.05, less uptake was observed in 661W compared to ARPE-19. This difference may be attributed to the fact that uptake rates are also specific to each cell type [31]. It is not surprising that photoreceptor cells have a lower uptake rate compared to ARPE-19 cells, because RPE cells have a high rate of phagocytosis, in fact one of their functions is to phagocytose photoreceptor outer segments [32]. The reduced uptake at $4^{\circ} \mathrm{C}$ suggested that the SLPs mainly enter the cells via endocytosis, as the energy dependent endocytosis will be largely inhibited at this temperature [33-35]. Finally, we demonstrated that SLPs can release their cargo after being internalized by the cells. This result highlights that the newly developed DDS potential is appropriate for encapsulation of hydrophilic drugs and for their release into the target cells.

The in vitro cytotoxicity studies provided the insight that the two retinal cell lines were differently affected by our DDS. This is in agreement with previous studies reporting that distinctive cell physiology, proliferation rate, metabolic activity, membrane, and phagocytosis characteristics are responsible for the different sensitivity to external factors $[36,37]$. Physicochemical elements of nanoparticles can also affect the cytotoxicity of cells [37]. Specifically, SLP.05 and SLP.06, explaining distinct shell composition, were differently toxic to retinal cells. Our results suggest that the DDS shell type composition and concentration must be carefully tailored for the specific cell type that needs to be targeted.

While the relatively simple cell culture environment yielded interesting data, a full drug/DDS efficacy testing will likely require more complex test systems. More advanced in vitro tests using organotypic retinal explant cultures, in which the normal histotypic context of the retina is preserved [38], or in vivo injections will further characterize the suitability of the new SLPs for the delivery to the retina. Nevertheless, based on our formulation development and initial validation studies, this DDS appears to be suitable for intraocular administration of small hydrophilic compound for the treatment of retinal diseases.

\section{Conclusion}

The current work demonstrates the successful development of a SLP formulation 
capable of holding small hydrophilic cargo and deliver it to retinal cells in vitro. Hydrogel core plays an important role in the SLP ability to encapsulate the cargo efficiently. Also, the type of hydrophobic polymer used for the composite shell affect SLP surface charge as well as polydispersity. The physicochemical properties of the DDS developed using RhoB were retained when the neuroprotective cGMPS analogue (i.e., CNo3) was used as a cargo. The in vitro validation study demonstrated that our DDS can be uptaken by model retinal cell lines (i.e., ARPE-19 and 661W). Flow cytometry study showed that the particle shell composition and cell type may influence the rate of particle uptake. Based on these developments and initial validation studies, our work may open new perspectives for developing a treatment for retinal diseases using small hydrophilic cargo. While the current results are promising, in vivo studies need to be performed to demonstrate the clinical relevance.

\section{Future perspective}

Delivering drug to the retina is challenging, especially when an active molecule of interest is small and hydrophilic in nature. To avoid fast clearance and improve drug internalization into targeted cells cytoplasm, a drug delivery system could be important during the drug formulation development. Our work demonstrates that SLP containing hydrogel core and composite shell has potential in solving these challenges. Based on our study in small hydrophilic molecules encapsulation and its subsequent release into cytoplasm, we surmise that our drug delivery system could potentially be useful for another small hydrophilic cargo (e.g., DNA) for the development of retinal disease treatment. This drug delivery system may also be adapted for biomarker development that uses small hydrophilic molecule which need to be internalized by the cells.

\section{Summary points}

- A solid lipid nanoparticle (SLP) having a hydrogel core and composite shell was formulated using modified water-in-oil-in-water emulsion technique to encapsulate and deliver small hydrophilic cargo to retinal cells.

- Introducing hydrogel core based on thermo-responsive gelling polymer to SLP formulation significantly enhances small hydrophilic cargo encapsulation efficiency, which can reach up to two-fold improvement compared to conventional SLP formulation with only aqueous core.

- Even higher encapsulation efficiency of small hydrophilic cargo was observed when the cargo has low solubility in organic solvent.

- Having hydrophobic polyester in the solid lipid shell formulation may improves particle polydispersity and modulate nanoparticle surface charge based on the type of polymer used.

- The SLP formulated is capable to improve small hydrophilic cargo uptake into retinal cell lines in vitro.

- Most importantly, as a nanoparticulate delivery system, co-encapsulation of tracer (ANTS) and quencher (DPX) molecules showed that the SLP can successfully be broken down after uptake and release its hydrophilic cargo into cytoplasm.

\section{Acknowledgements and funding information}

We thank Prof Gareth R. Williams from University College London for his assistance in TEM images. We thank Dr. M. Montanari for technical support on flow cytometry. This study was supported by grants from the European Union (transMed; H2020-MSCA-ITN765441) and the Charlotte and Tistou Kerstan Foundation. 


\section{References}

1 Lechner J, O'Leary OE, Stitt AW. The pathology associated with diabetic retinopathy. Vision Res. 139, 7-14 (2017).

2 Mitchell P, Liew G, Gopinath B, Wong TY. Age-related macular degeneration. Lancet 392(10153), 1147-1159 (2018).

3 Swaroop A, Sieving PA. The golden era of ocular disease gene discovery: Race to the finish. Clin. Genet. 84(2), 99-101 (2013).

4 Chakravarthy U, Biundo E, Saka RO, Fasser C, Bourne R, Little JA. The Economic Impact of Blindness in Europe. Ophthalmic Epidemiol. 24(4), 239-247 (2017).

5 Peeters L, Sanders NN, Braeckmans K et al. Vitreous: A Barrier to Nonviral Ocular Gene Therapy. Investig. Opthalmology Vis. Sci. 46(10), 3553 (2005).

$6 \mathrm{Xu}$ Q, Boylan NJ, Suk JS et al. Nanoparticle diffusion in, and microrheology of, the bovine vitreous ex vivo. J. Control. Release 167(1), 76-84 (2013).

$7 \quad$ Tavakoli S, Kari OK, Turunen T et al. Diffusion and Protein Corona Formation of Lipid-Based Nanoparticles in the Vitreous Humor: Profiling and Pharmacokinetic Considerations. Mol. Pharm. 18(2), 699-713 (2021).

8 del Amo EM, Rimpelä AK, Heikkinen E et al. Pharmacokinetic aspects of retinal drug delivery. Prog. Retin. Eye Res. 57, 134-185 (2017).

9 Himawan E, Ekström P, Buzgo M et al. Drug delivery to retinal photoreceptors. Drug Discov. Today 24(8), 1637-1643 (2019).

10 Apaolaza PS, del Pozo-Rodríguez A, Solinís MA et al. Structural recovery of the retina in a retinoschisin-deficient mouse after gene replacement therapy by solid lipid nanoparticles. Biomaterials 90, 40-49 (2016).

11 Bisht R, Mandal A, Jaiswal JK, Rupenthal ID. Nanocarrier mediated retinal drug delivery: overcoming ocular barriers to treat posterior eye diseases. Wiley Interdiscip. Rev. Nanomedicine Nanobiotechnology 10(2), 1-21 (2018).

12 Gorantla S, Rapalli VK, Waghule T et al. Nanocarriers for ocular drug delivery: Current status and translational opportunity. RSC Adv. 10(46), 27835-27855 (2020).

13 Gallego I, Villate-Beitia I, Martínez-Navarrete G et al. Non-viral vectors based on cationic niosomes and minicircle DNA technology enhance gene delivery efficiency for biomedical applications in retinal disorders. Nanomedicine Nanotechnology, Biol. Med. 17, 308-318 (2019).

Tolone A, Belhadj S, Rentsch A, Schwede F, Paquet-Durand F. The cGMP pathway and inherited photoreceptor degeneration: Targets, compounds, and biomarkers. Genes (Basel). 10(6), 1-16 (2019). Kaiser JM, Imai H, Haakenson JK et al. Nanoliposomal minocycline for ocular drug delivery. Nanomedicine Nanotechnology, Biol. Med. 9(1), 130-140 (2013).

16 Chaharband F, Daftarian N, Kanavi MR et al. Trimethyl chitosan-hyaluronic acid nano-polyplexes for intravitreal VEGFR-2 siRNA delivery: Formulation and in vivo efficacy evaluation. Nanomedicine Nanotechnology, Biol. Med. 26, 102181 (2020).

17 Li Q, Li X, Zhao C. Strategies to Obtain Encapsulation and Controlled Release of Small Hydrophilic Molecules. Front. Bioeng. Biotechnol. 8(May), 1-6 (2020).

18 Zariwala MG, Bendre H, Markiv A et al. Hydrophobically modified chitosan nanoliposomes for intestinal drug delivery. Int. J. Nanomedicine 13, 5837-5848 (2018).

19 Khalkhali M, Mohammadinejad S, Khoeini F, Rostamizadeh K. Vesicle-like structure of lipid-based nanoparticles as drug delivery system revealed by molecular dynamics simulations. Int. J. Pharm. 559, 173-181 (2019).

20 Severino P, Pinho SC, Souto EB, Santana MHA. Polymorphism, crystallinity and hydrophiliclipophilic balance of stearic acid and stearic acid-capric/caprylic triglyceride matrices for production of stable nanoparticles. Colloids Surfaces B Biointerfaces 86(1), 125-130 (2011).

21 Yang R, Gao RC, Cai CF et al. Preparation of gel-core-solid lipid nanoparticle: A novel way to improve the encapsulation of protein and peptide. Chem. Pharm. Bull. 58(9), 1195-1202 (2010).

Chen C, Zhu X, Dou Y et al. Exendin-4 loaded nanoparticles with a lipid shell and aqueous core containing micelles for enhanced intestinal absorption. J. Biomed. Nanotechnol. 11(5), 865-876 (2015). 
Xu Y, Zheng Y, Wu L, Zhu X, Zhang Z, Huang Y. Novel Solid Lipid Nanoparticle with Endosomal Escape Function for Oral Delivery of Insulin. ACS Appl. Mater. Interfaces 10(11), 9315-9324 (2018). Vighi E, Trifunovic D, Veiga-Crespo P et al. Combination of cGMP analogue and drug delivery system provides functional protection in hereditary retinal degeneration. Proc. Natl. Acad. Sci. U. S. A. 115(13), E2997-E3006 (2018). Martens TF, Remaut K, Demeester J, De Smedt SC, Braeckmans K. Intracellular delivery of nanomaterials: How to catch endosomal escape in the act. Nano Today 9(3), 344-364 (2014). Dunn KC, Aotaki-Keen AE, Putkey FR, Hjelmeland LM. ARPE-19, A Human Retinal Pigment Epithelial Cell Line with Differentiated Properties. Exp. Eye Res. 62(2), 155-170 (1996). Tan E, Ding X-Q, Saadi A, Agarwal N, Naash MI, Al-Ubaidi MR. Expression of Cone-PhotoreceptorSpecific Antigens in a Cell Line Derived from Retinal Tumors in Transgenic Mice. Investig. Opthalmology Vis. Sci. 45(3), 764 (2004). polystyrene nanoparticles and factors affecting the uptake: Relevance for drug delivery systems. Eur. J. Cell Biol. 93(8-9), 323-337 (2014). Yang ST, Zaitseva E, Chernomordik L V., Melikov K. Cell-penetrating peptide induces leaky fusion of liposomes containing late endosome-specific anionic lipid. Biophys. J. 99(8), 2525-2533 (2010). He C, Hu Y, Yin L, Tang C, Yin C. Effects of particle size and surface charge on cellular uptake and biodistribution of polymeric nanoparticles. Biomaterials 31(13), 3657-3666 (2010). Kumari S, MG S, Mayor S. Endocytosis unplugged: multiple ways to enter the cell. Cell Res. 20(3), 256-275 (2010).

32 Bertolotti E, Neri A, Camparini M, Macaluso C, Marigo V. Stem cells as source for retinal pigment epithelium transplantation. Prog. Retin. Eye Res. 42, 130-144 (2014).

33 Behzadi S, Serpooshan V, Tao W et al. Cellular uptake of nanoparticles: journey inside the cell. Chem. Soc. Rev. 46(14), 4218-4244 (2017).

34 Palocci C, Valletta A, Chronopoulou L et al. Endocytic pathways involved in PLGA nanoparticle uptake by grapevine cells and role of cell wall and membrane in size selection. Plant Cell Rep. 36(12), 1917-1928 (2017). Surface Modification Modulates Cell Internalization and Improves Chemotoxic Treatment in an Oral Carcinoma Cell Line. Nanomaterials 9(3), 464 (2019). nanoparticles. Nanomedicine 6(5), 929-941 (2011). Foroozandeh P, Aziz AA. Insight into Cellular Uptake and Intracellular Trafficking of Nanoparticles. Nanoscale Res. Lett. 13(1), 339 (2018). Cultivation of Organotypic Mouse Retina Explants with Intact Retinal Pigment Epithelium. J. Vis. Exp. (165) (2020). 